\title{
Analysing by decade, testosterone undecanoat depot injectable does not increases prostate volume. Study during up to 7 years on hypogonadic patients.
}

Matei Pisoschi1 ${ }^{1}$, Mara Carşote ${ }^{2}$, Cătălina Poiană $^{2}$, Cristina Daniela Staicu $^{3}$, Dan Perețianu ${ }^{3}$ 1. Hospital "T. Burghele"; 2. Inst. Endocrinology "Parhon", 3. SCM "Povernei", Bucharest - Romania Aim. Re-Analyzing the effect on prostatic volume of injectable testosterone undecanoat depot (TUD) in hypogonadic patients without prostatic cancer.

Material\&Method. A. Patients at onset 210 men with hypogonadism (median age: 61.5 y). B. Distribution: by decade (no): 4,

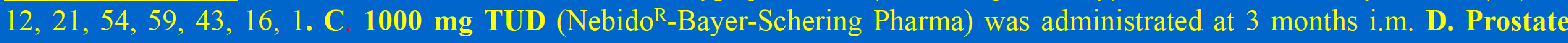
volume (PV) appreciated by per-abdominal ultrasound: $3,5 \mathrm{MHz}$ probe, elliptical volume ( $\left.\mathrm{cm}^{3}\right)$, Aloka 550. E. Time of analysis: before starting testosterone (T0), after $1 / 2$ (T1), 3 (T2), 6 months (T3), 1 (T4), 2 (T5), 3 (T6), 4 (T7), 5 (T8), 6 (T9) and 7 years (T10). F. Maximum increment from P0 was noted $\Delta \mathrm{M} \%$. Average increment was noted $\Delta$ A $\%$. G. Statistical analysis by Student test $(\mathrm{p})$

Results. I. All average data of prostatic volume, maximum increment $(\Delta \mathrm{M} \%)$ per decade, including the moment ( $\Delta \mathrm{M} \%$ at $)$ and average increment ( $\triangle \mathrm{A} \%$ )-tabulatecd. PV at T0 increases with age, from minimum 15.38 (19-29y) to maximum 47,41 (80-89y), $\mathrm{p}=0.0007$. III. Considering all observations (some at 5/6/7 years), TUD did not increase PV significantly (in fact the average of

\begin{tabular}{|c|c|c|c|c|c|c|c|c|c|c|c|c|c|c|c|c|c|c|c|c|c|c|c|}
\hline Decade & \begin{tabular}{|c} 
Age \\
at \\
onset \\
Av \\
\end{tabular} & $\begin{array}{c}\text { PV } \\
\mathbf{0}\end{array}$ & No & $\begin{array}{l}\text { PV } \\
14 d\end{array}$ & No & $\begin{array}{l}\text { PV } \\
3 m\end{array}$ & No & $\begin{array}{l}\text { PV } \\
6 \mathrm{~m}\end{array}$ & No & $\begin{array}{c}\mathrm{PV} \\
12 \mathrm{~m}\end{array}$ & No & $\begin{array}{l}\text { PV } \\
2 Y\end{array}$ & No & $\begin{array}{l}\text { PV } \\
3 Y\end{array}$ & No & $\begin{array}{l}\text { PV } \\
4 Y\end{array}$ & No & $\begin{array}{l}\text { PV } \\
5 Y\end{array}$ & No & $\begin{array}{l}\text { PV } \\
6 Y\end{array}$ & No & $\begin{array}{l}\text { PV- } \\
7 Y\end{array}$ & No \\
\hline & & \multicolumn{2}{|c|}{ T0 } & \multicolumn{2}{|c|}{$\mathrm{T} 1$} & \multicolumn{2}{|c|}{$\mathrm{T} 2$} & \multicolumn{2}{|c|}{ T3 } & \multicolumn{2}{|c|}{$\mathrm{T} 4$} & \multicolumn{2}{|c|}{ T5 } & \multicolumn{2}{|c|}{ T6 } & \multicolumn{2}{|c|}{$\mathrm{T} 7$} & \multicolumn{2}{|c|}{$\mathrm{T} 8$} & \multicolumn{2}{|c|}{ T9 } & \multicolumn{2}{|c|}{$\mathrm{T} 10$} \\
\hline $18-29$ & 23.00 & 15.38 & 4 & 13.75 & 4 & 15.50 & 4 & 15.50 & 4 & 17.13 & 4 & 15.50 & 4 & 17.33 & 3 & 14.00 & 2 & 17.00 & 1 & 16.00 & 1 & & \\
\hline $30-39$ & 35.92 & 17.83 & 12 & 17.29 & 12 & 20.75 & 6 & 22.70 & 5 & 21.75 & 4 & 25.50 & 2 & 24.50 & 2 & 24.50 & 2 & 18.00 & 2 & 18.50 & 2 & 17.00 & 2 \\
\hline $40-49$ & 46.00 & 25.33 & 21 & 26.33 & 21 & 27.30 & 15 & 28.73 & 14 & 29.00 & 12 & 29.28 & 9 & 33.50 & 6 & 46.50 & 4 & 47.17 & 3 & 69.00 & 2 & 22.00 & 1 \\
\hline $50-59$ & 55.17 & 28.31 & 54 & 28.02 & 54 & 27.49 & 48 & 29.60 & 37 & 29.72 & 31 & 30.55 & 20 & 29.97 & 17 & 31.61 & 14 & 29.45 & 10 & 25.63 & 4 & 22.00 & 1 \\
\hline $60-69$ & 64.49 & 34.99 & 59 & 33.60 & 59 & 34.84 & 44 & 32.08 & 33 & 32.91 & 28 & 33.60 & 21 & 37.40 & 10 & 39.44 & 10 & 39.93 & 7 & 26.33 & 3 & 0 & 0 \\
\hline $70-79$ & 74.98 & 45.12 & 43 & 45.03 & 43 & 44.49 & 34 & 42.54 & 28 & 40.03 & 21 & 43.71 & 13 & 41.88 & 9 & 46.08 & 6 & 60.67 & 3 & & & & \\
\hline $80-89$ & 83.41 & 47.41 & 16 & 43.31 & 16 & 46.00 & 14 & 46.40 & 11 & 50.83 & 9 & 61.07 & 7 & 81.00 & 4 & 78.75 & 2 & 74.00 & 2 & & & & \\
\hline$>90$ & 96.00 & 32.00 & 1 & 34.00 & 1 & 40.00 & 1 & & & & & & & & & & & & & & & & \\
\hline Total & & & 210 & & 210 & & 166 & & 132 & & 109 & & 76 & & 51 & & 40 & & 28 & & 12 & & 4 \\
\hline
\end{tabular}

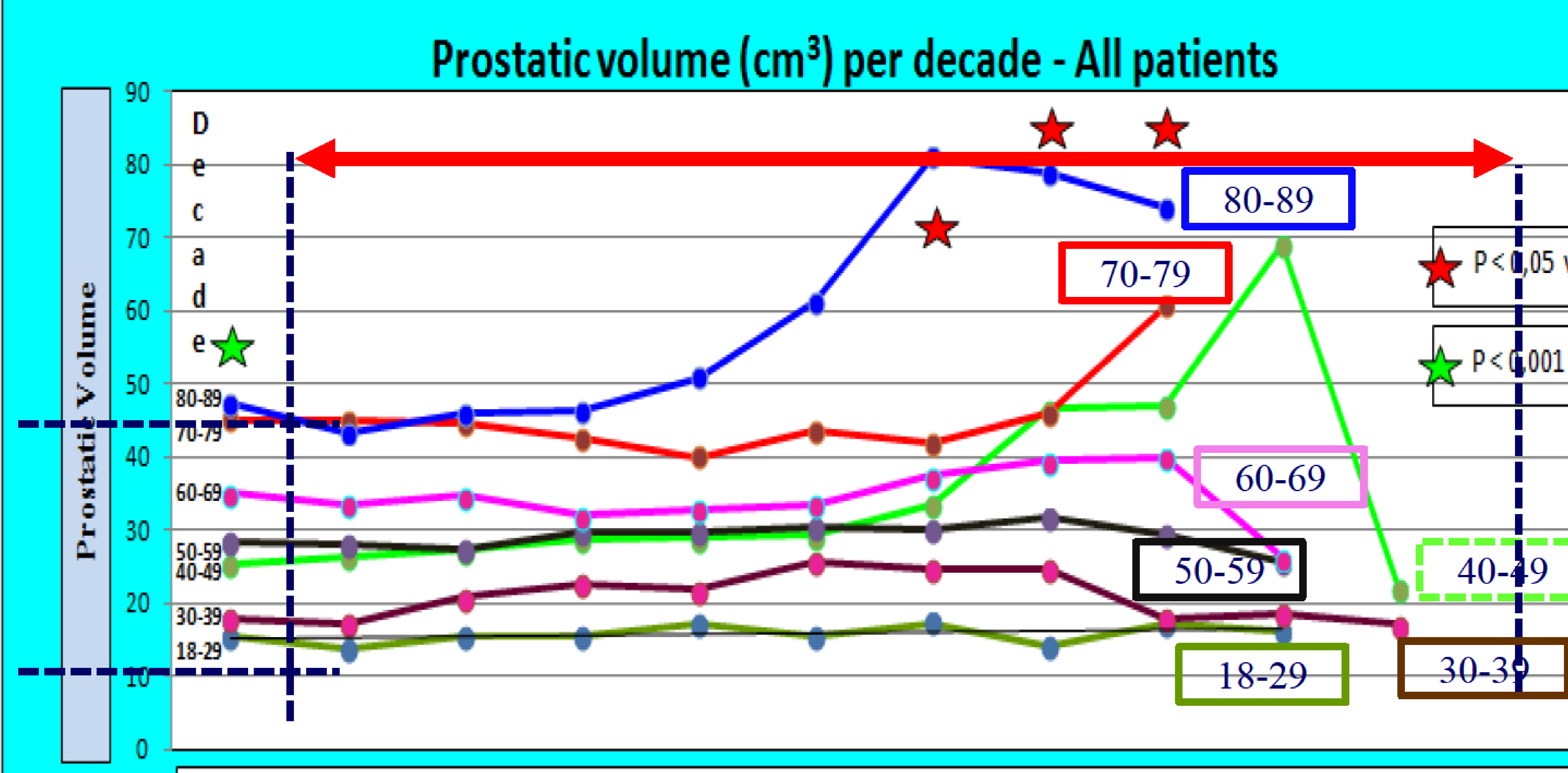

Before 14 days 3 months 6 months 1 year 2 years 3 years 4 years 5 years 6 years 7 years treatment increment in all patients was negative $12,54 \%)$. D M \% per decade: 45,95; 20; 40,35; 42,$5 ; 45.45 ; 64.41 ; 39,56 ; 20$ IV. Inside a specific decade no significant increased in PV was registered: all $\mathrm{p}>0,05$; exception: 80-89 decade, $\mathrm{p}=0,002$, at $3-5 \mathrm{y}$. V. In some patients, especially from 50-79 years, TUD could decrease slightly prostatic volume.

\begin{tabular}{|c|c|c|c|c|}
\hline Decade & $\Delta \mathrm{M} \%$ & $\Delta \mathrm{M} \%$ at & $\begin{array}{c}\mathrm{P}(\mathrm{T} \text { test }) \mathrm{T} 0 \\
\mathrm{v} \mathrm{T} \max \end{array}$ & $\Delta \mathrm{A} \%$ \\
\hline $18-29$ & 45.95 & $\mathrm{~T} 4$ & 0.68 & -3.72 \\
\hline $30-39$ & 20.00 & $\mathrm{~T} 3$ & 0.26 & -11.42 \\
\hline $40-49$ & 40.35 & $\mathrm{~T} 2$ & 0.68 & 2.62 \\
\hline $50-59$ & 42.50 & $\mathrm{~T} 2$ & 0.72 & -12.76 \\
\hline $60-69$ & 45.45 & $\mathrm{~T} 1$ & 0.64 & -14.95 \\
\hline $70-79$ & 64.41 & $\mathrm{~T} 2$ & 0.92 & -34.25 \\
\hline $80-89$ & 39.56 & $\mathrm{~T} 6$ & 0.22 & -0.02 \\
\hline$>90$ & 20.00 & $\mathrm{~T} 2$ & & 12.94 \\
\hline
\end{tabular}

Conclusions. Considering the risk for prostate (in elderly), testosterone undecanoat 1000 mg depot injectable is a safe treatment, even after 3 to 7 years of administration. Precautions should be accorded to men over $70 \mathrm{y}$, after 4 years and 80 y old, after 6 months administration. 